\title{
Negative Staining Technique of Heine for the Detection of Cryptosporidium spp.: A Fast and Simple Screening Technique
}

\author{
Idzi Potters* and Marjan Van Esbroeck
}

\author{
Institute of Tropical Medicine, Department of Clinical Sciences, Nationalestraat 155, 2000 Antwerp, Belgium
}

\begin{abstract}
Although inexpensive and easy to perform, the negative staining technique of Heine for the detection of Cryptosporidium spp. has been largely neglected. The lack of familiarity with the negative staining technique, the misconception that a phase-contrast microscope is indispensible and inferior results due to Köhler-illumination, are possible explanations for its low popularity. While the modified Ziehl-Neelsen staining technique is still considered the Gold Standard for the detection of Cryptosporidium spp., the negative staining technique of Heine should be reconsidered as the screening technique of first choice. Advantages of the negative staining technique of Heine over the modified Ziehl-Neelsen staining are discussed.
\end{abstract}

Keywords: Cryptosporidium, Heine, Ziehl-Neelsen, Köhler, Phase-contrast.

Cryptosporidium spp. are intracellular, extracytoplasmic coccidian parasites of the intestinal tract and have been recognized as a pathogen in humans since 1976 [1]. This parasite is transmitted in several ways including waterborne, person-to-person, zoonotic, foodborne and possibly airborne $[2,3]$. Cryptosporidium spp. infect not only humans, but also other animals. In man, cryptosporidiosis usually causes a violent attack of profuse, watery diarrhea with abdominal pain, malaise, nausea, vomiting and fever [4,5]. In healthy subjects, cryptosporidiosis is self-limiting and symptoms only persist for some days or weeks. In immunocompromised subjects and children, the illness is known to be more severe and even life-threatening [4-9]. Detection rates in industrialized countries are between $1 \%$ and 3\%. Mean prevalence rates in developing countries can reach $5 \%$ to $10 \%$ [5]. In neonates of some mammals, such as ruminants, the severity of infection, as well as the prevalence of Cryptosporidium spp., can be high [10]. Cryptosporidiosis is considered one of the most common causes of neonatal diarrhea in cattle [11]; it reduces growth rate of calves and sheep, impairs feed conversions and reduces milk production [10,12-14]. Mortality rates, in young calves, can reach $35 \%$ [15] resulting in significant economic loss.

A variety of methods is available for the detection of Cryptosporidium spp., including microscopic, immunological and molecular techniques. Microscopic detection is based on finding the environmentally and chemically resistant oocysts $[16,17]$ in faecal samples. The oocysts however cannot be positively identified in wet mounts. For this reason, the faecal sample is spread out on a glass slide and stained. If needed, samples can be concentrated using either flotation or sedimentation techniques prior to staining [18]. Immuno-

*Address correspondence to this author at the Institute of Tropical Medicine, Department of Clinical Sciences, Nationalestraat 155, 2000 Antwerp, Belgium; Tel: 0032-(0)3-247.64.38; Fax: 0032-(0)3-247.64.40;

E-mail: ipotters@itg.be logical and molecular techniques are more complex and costly, making them less useful methods for screening, especially in resource-poor settings. However, they have usually better sensitivities and specificities [19,20].

As microscopy is a rapid, cost-effective and reliable diagnostic tool, this paper suggests the negative staining technique of Heine as an easy, inexpensive and efficient way of screening for Cryptosporidium spp. This technique, which uses undiluted carbol-fuchsine, is compared to the modified acid fast Ziehl-Neelsen staining, the Gold Standard for the detection of Cryptosporidium spp. These two techniques have been selected for comparison as other techniques may require less common staining products (e.g. safranine) or even fluorescence microscopes (e.g. auramine-phenol staining). Minor modifications are made to improve the sensitivity and feasibility of the negative staining technique of Heine.

The modified Ziehl-Neelsen staining is classically performed by staining a methanol fixed thin smear of faecal material with undiluted carbol-fuchsine solution for at least 15 minutes. Subsequently, the slide is rinsed in tap water and placed in an acid-alcohol solution to remove the stain, while acid-fast structures will resist to the acid-alcohol's destaining action. After rinsing again, the slide is placed for a short period of time in a counter-staining product, such as methylene blue, providing contrast between background material and acid-fast structures. The slide is rinsed once more and after the slide has been air-dried, it can be examined using x 10 eyepieces and an oil-immersion objective of x100 magnification $[21,22]$. Cryptosporidium oocysts will appear as pinkstained, round to oval structures of about 3 to $6 \mu \mathrm{m}$ in diameter, containing distinct internal structures (Fig. 1A). The modified Ziehl-Neelsen staining is a time-consuming procedure (about 30 to 45 minutes), which requires intensive training and experience to interpret the results [23-26]. A common problem is distinguishing Cryptosporidium oocysts from other elements, such as moulds and yeast $[18,26]$. 


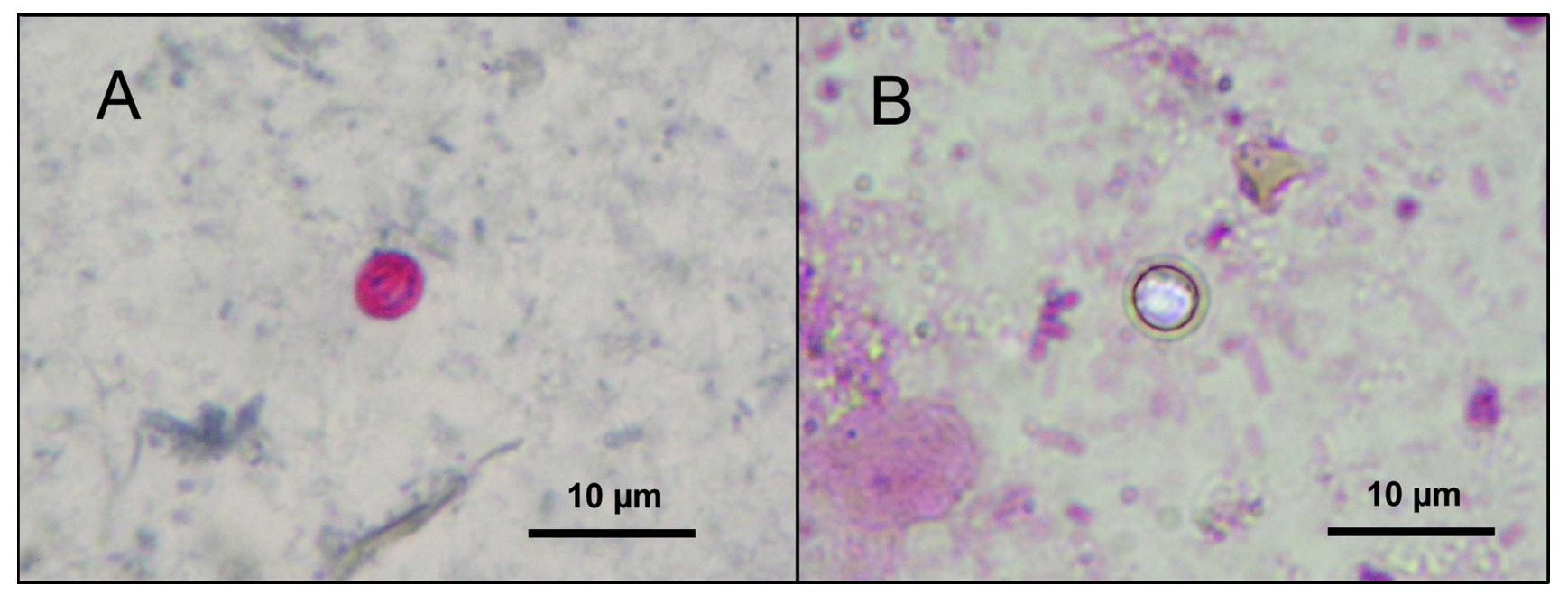

Fig. (1). Oocyst of Cryptosporidium spp., stained by the modified Ziehl-Neelsen staining technique (A) and stained by the negative staining technique of Heine (B).

These "pseudo-Cryptosporidia" can be ruled out based on their dimensions [18]. Although the modified Ziehl-Neelsen staining remains the Gold Standard for the detection of Cryptosporidium spp., it is claimed to lack sensitivity [26] and specificity $[18,26]$. The lack of specificity could be resolved by lowering the sensitivity of the test. For instance a sample could be considered positive if five oocysts or more were observed, causing low-level shedding of oocysts to become interpreted as negative samples [27]. This extra loss of sensitivity in turn could be resolved by using repeated stool sample examinations on consecutive days [28]. The modified Ziehl-Neelsen staining is a low cost technique (about 0,15 US\$ per sample), while the cost for PCR-based diagnosis is considerably higher (about 7,60 US\$ per sample) [29]. In addition, the modified Ziehl-Neelsen staining technique provides a permanent stain, making it possible to send doubtful or scanty positive slides to a reference laboratory for confirmation.

For the negative staining technique of Heine, a small amount of faecal matter is mixed with an equal amount of undiluted carbol-fuchsine solution on a microscope slide. A thin smear is prepared, allowed to air dry and examined using x10 eyepieces and an oil-immersion objective of x50 or x100 magnification [30]. Cryptosporidium oocysts appear as unstained, strongly refractive, round to oval structures of about 3 to $6 \mu \mathrm{m}$ in diameter. Internal structures are slightly visible as darker specks inside the oocyst (Fig. 1B). The slides should be examined within 15 minutes after they have been air-dried [30]. This time-lapse can be prolonged to 30 minutes by using samples which have been fixed in $10 \%$ formalin, prior to staining (Fig. 2). If the slide is not examined in 15 to 30 minutes the oocysts will dry out and become less visible (Fig. 2G, $\mathbf{H}$ and $\mathbf{I}$ ). According to some workers a phase-contrast microscope is indispensable [26]. Our experience is that phase-contrast indeed raises specificity and sensitivity (Fig. 2C, F and I), but oocysts can still be recog- nized without phase-contrast. To improve the sensitivity of the negative staining technique, we recommend adding a drop of oil on the slide and covering it with a coverslip. By doing so, the preparation can be observed using x10 eyepieces and a "dry" objective of x40 magnification [30]. This modification does not work if the oil is replaced with physiological saline. Most striking is that the visibility of oocysts drops significantly when the microscope is set to Köhlerillumination in a brightfield position (Fig. 2B, $\mathbf{E}$ and $\mathbf{H}$ ). Köhler-illumination allows only the straight rays of light to pass through the optical system, while blocking all scattered rays of light. This will cause the negatively stained oocysts to lose their refractivity. Lowering the microscope condenser when working in brightfield position avoids this problem.

The lack of familiarity with the negative staining technique of Heine, the misconception that a phase-contrast microscope is indispensible, and inferior results caused by Köhler-illumination, are possible explanations for the low popularity of this staining technique. Compared to the modified Ziehl-Neelsen staining, the negative staining technique is simpler and costs less to implement, as it uses only one stain. The time needed for this staining is less than half the time needed for the modified Ziehl-Neelsen staining and the sensitivity can be increased by using phase-contrast microscopy or examination at $\mathrm{x} 400$ magnification, both of which are not possible with the modified Ziehl-Neelsen staining technique. We therefore recommend the negative staining technique of Heine as the first choice for screening of slides for Cryptosporidium spp. Doubtful or equivocally positive samples can be confirmed, using the modified Ziehl-Neelsen staining or other, more sophisticated techniques.

\section{ACKNOWLEDGEMENTS}

Special thanks go to Dr. Lieselotte Cnops (Institute of Tropical Medicine, Antwerp, Belgium), to Bruno Levecke (Faculty of Veterinary Medicine, Ghent University, Merel- 


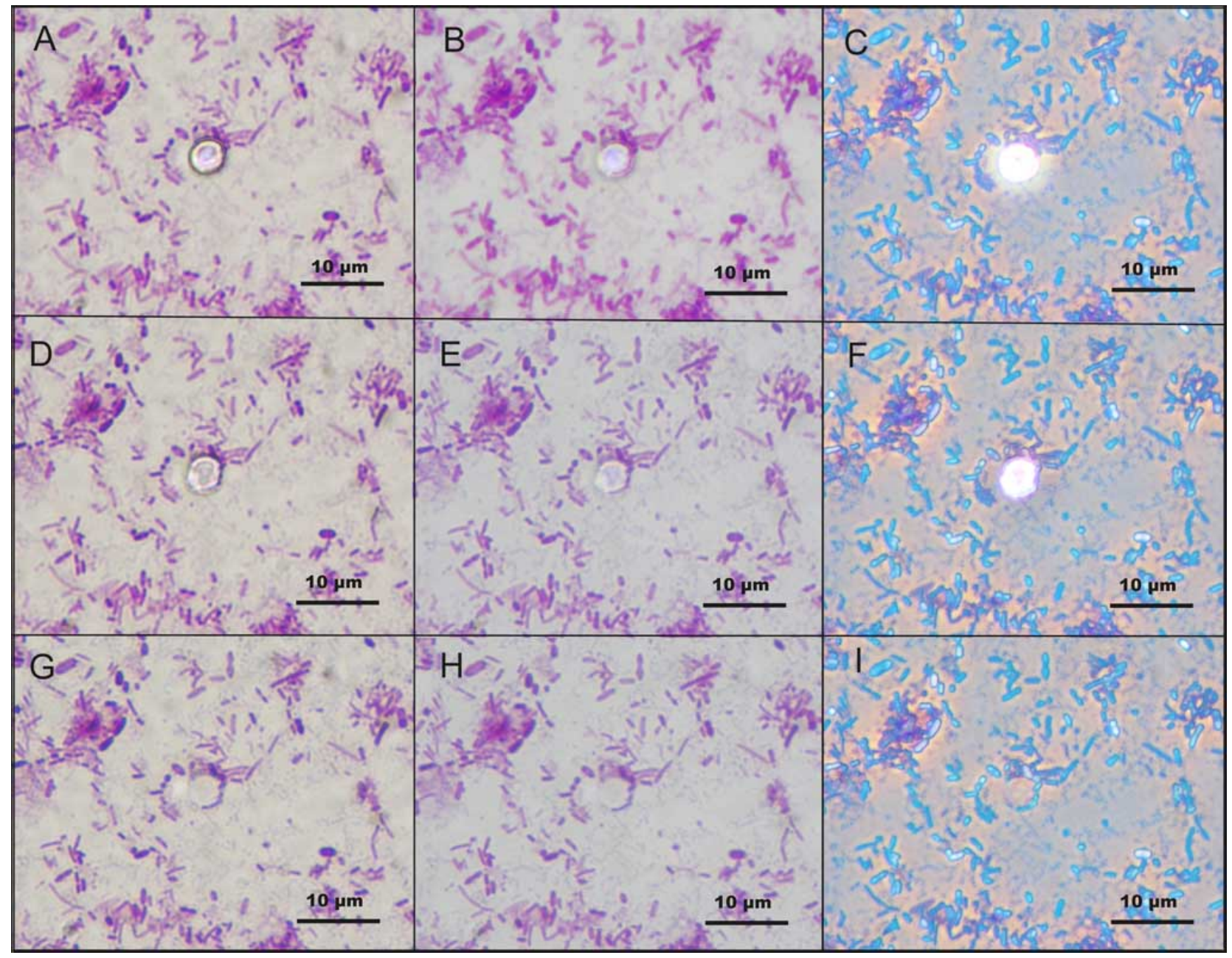

Fig. (2). Oocyst of Cryptosporidium spp., fixed with $10 \%$ formaline solution, stained with the negative staining technique of Heine, immediately after the preparation has been air-dried (A, B and $\mathbf{C}), 35$ minutes after the preparation has been air-dried $(\mathbf{D}, \mathbf{E}$ and $\mathbf{F})$ and 50 minutes after the preparation has been air-dried $(\mathbf{G}, \mathbf{H}$ and $\mathbf{I})$. Each time as seen with a x100 oil-immersion lens, without Köhler illumination in brightfield position (A, D and $\mathbf{G})$, with Köhler illumination in brightfield position $(\mathbf{B}, \mathbf{E}$ and $\mathbf{H})$ and with phase-contrast $(\mathbf{C}, \mathbf{F}$ and $\mathbf{I})$.

beke, Belgium) and to Dr. Masoud Dara for reviewing the manuscript and for their useful suggestions and corrections.

\section{REFERENCES}

[1] Nime FA, Burek JD, Page DL, Holscher MA, Yardley JH. Acute enterocolitis in a human being infected with the protozoan Cryptosporidium. Gastro-enterology 1976; 70: 592-8.

[2] Thompson RCA, Olson ME, Zhu G, Enomoto S, Abrahamsen MS, Hijjawi NS. Cryptosporidium and cryptosporidiosis. Adv Parasitol 2005; 59: 77-158.

[3] Cacciò SM, Thompson RCA, McLauchlin J, Smith HV. Unravelling Cryptosporidium and Giardia epidemiology. Trends Parasitol 2005; $21: 430-7$

[4] Casemore DP, Sands RL, Curry A. Cryptosporidium species a "new" human pathogen. J Clin Pathol 1985; 38: 1321-36.

[5] Current WL, Garcia LS. Cryptosporidiosis. Clin Microbiol Rev 1991; 4: 325-58

[6] Current WL, Reese NC, Ernst JV, Bailey WS, Heyman MB, Weinstein WM. Human cryptosporidiosis in immunocompetent and immunodeficient persons: studies of an outbreak and experimental transmission. N Engl J Med 1983; 308: 1252-7.
[7] Nannini EC, Okhuysen PC. HIV1 and the gut in the era of highly active antiretroviral therapy. Curr Gastroenterol Rep 2002; 4: 3928.

[8] Tumwine JK, Kekitiinwa A, Bakeera-Kitaka S, Ndeezi G, Downing R, Feng X, Akiyoshi DE, Tzipori S. Cryptosporidiosis and microsporidiosis in Ugandan children with persistent diarrhea with and without concurrent infection with the human immunodeficiency virus. Am J Trop Med Hyg 2005; 73: 921-5.

[9] Wolska-Kusnierz B, Bajer A, Caccio S, Heropolitanska-Pliszka E Bernatowska E, Socha P, van Dongen J, Bednarska M, Paziewska A, Sinski E. Cryptosporidium infection in patients with primary immunodeficiencies. J Pediatr Gastroenterol Nutr 2007; 45: 45864.

[10] Azami M. Prevalence of Cryptosporidium infection in cattle in Isfahan, Iran. J Eukaryot Microbiol 2007; 54: 100-2.

[11] Angus KW. Cryptosporidiosis in ruminants. In: Dubey JP, Speer CA, Fayer R, Eds. Cryptosporidiosis of man and animals, CRC Press Inc., Boca Raton 1990; pp.83-104.

[12] Esteban E, Anderson BC. Cryptosporidium muris: prevalence, persistency and detrimental effect on milk production in a drylot dairy. J Dairy Sci 1995; 78: 1068-72.

[13] Anderson BC. Cryptosporidiosis in bovine and human health. J Dairy Sci 1998; 81: 3036-41. 
[14] Alonso-Fresán MU, García-Álvarez A, Salazar-García F, VázquezChagoyán JC, Pescador-Salas N, Saltijeral-Oaxaca J. Prevalence of Cryptosporidium spp. in asymptomatic sheep in family flocks from Mexico State. J Vet Med B 2005; 52: 482-3.

[15] Singh BB, Sharma R, Kumar H, Banga HS, Aulakh RS, Gill JP, Sharma JK. Prevalence of Cryptosporidium parvum infection in Punjab (India) and its association with diarrhea in neonatal dairy calves. Vet Parasitol 2006; 140: 162-5.

[16] O'Donoghue PJ. Cryptosporidium and cryptosporidiosis in man and animals. Int J Parasitol 1995; 25: 139-95.

[17] Quilez J, Sanchez-Acedo C, Del-Cacho E, Clavel A, Causapé AC. Prevalence of Cryptosporidium and Giardia infections in cattle in Aragon (Northeastern Spain). Vet Parasitol 1996; 66: 139-46.

[18] Casemore DP. Laboratory methods for diagnosing cryptosporidiosis. J Clin Pathol 1991; 44: 445-51.

[19] Morgan UM, Pallant L, Dwyer BW, Forbes DA, Rich G, Thompson RCA. Comparison of PCR and microscopy for detection of Cryptosporidium parvum in human fecal specimens: clinical trial. J Clin Microbiol 1998; 36: 995-8.

[20] Siddons CA, Chapman PA, Rush BA. Evaluation of an enzyme immunoassay kit for detecting Cryptosporidium in faeces and environmental samples. J Clin Pathol 1992; 45: 479-82.

[21] Henriksen SA, Pohlenz JFL. Staining of cryptosporidia by a modified Ziehl-Neelsen technique. Acta Vet Scand 1981; 22: 594-6.

[22] Casemore DP, Armstrong M, Sands RL. Laboratory diagnosis of cryptosporidiosis. J Clin Pathol 1985; 38: 1337-41.

[23] Weber R, Bryan RT, Bishop HS, Wahlquist SP, Sullivan JJ, Juranek DD. Threshold of detection of Cryptosporidium oocysts in human stool specimens: evidence for low sensitivity of current diagnostic methods. J Clin Microbiol 1991; 29: 1323-7.

[24] Mtambo MM, Nash AS, Blewett DA, Wright S. Comparison of staining and concentration techniques for detection of Cryptosporidium oocysts in cat faecal specimens. Vet Parasitol 1992; 45: 49-57.

[25] Tee GH, Moody AH, Cooke AH, Chiodini PL. Comparison of techniques for detecting antigens of Giardia lamblia and Cryptosporidium parvum in faeces. J Clin Pathol 1993; 46: 555-8.

[26] Chartier C, Mallereau-Pellet MP, Mancassola R, Nussbaum D. Détection des oocystes de Cryptosporidium dans les fèces de caprins : comparaison entre un test d'agglutination au latex et trois autres techniques conventionnelles. Vet Res 2002; 33: 169-77.

[27] Maldonado-Camargo S, Atwill ER, Saltijeral-Oaxaca JA, HerreraAlonso LC. Prevalence of and risk factors for shedding of Cryptosporidium parvum in Holstein Freisian dairy calves in central Mexico. Prev Vet Med 1998; 36: 95-107.

[28] Brook EJ, Christley RM, French NP, Hart CA. Detection of Cryptosporidium oocysts in fresh and frozen cattle faeces: comparison of three methods. Lett Appl Microbiol 2008; 46: 26-31.

[29] Paul S, Chandra D, Tewari AK, Banerjee PS, Ray DD, Boral R, Rao JR. Comparative evaluation and economic assessment of coprological diagnostic methods and PCR for detection of Cryptosporidium spp. in bovines. Vet Parasitol 2009; 164: 291-5.

[30] Heine J. Eine einfache Nachweismethode für Kryptosporidien im Kot. Zbl Vet Med B 1982; 29: 324-7.

(c) Potters and Van Esbroeck; Licensee Bentham Open.

This is an open access article licensed under the terms of the Creative Commons Attribution Non-Commercial License (http://creativecommons.org/licenses/by-nc/3.0/) which permits unrestricted, non-commercial use, distribution and reproduction in any medium, provided the work is properly cited. 